KANAZAWA 96-10

July 1996

\title{
Three topics of monopole dynamics in abelian projected QCD *
}

\author{
Tsuneo Suzuki a , Yoshimi Matsubara $^{\text {b }}$, Shun-ichi Kitahara ${ }^{\text {c }}$, Shinji Ejiri ${ }^{\mathrm{a}}$, \\ Naoki Nakamura $^{a}$, Fumiyoshi Shoji ${ }^{a}$, Masafumi Sei ${ }^{a}$, Seikou Kato ${ }^{a}$ and Natsuko Arasaki ${ }^{a}$ \\ a Department of Physics, Kanazawa University, Kanazawa 920-11, Japan

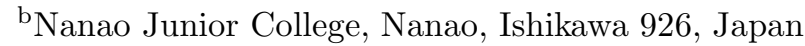 \\ c Jumonji University, Niiza, Saitama 352, Japan
}

Three topics about monopole dynamics after abelian projection are reported. The first is the new and detailed analyses of $S U(2)$ monopole action obtained after the block-spin transformation on the dual lattice. The $b=n a(\beta)$ dependence for all couplings are well fitted with a universal curve. The distance dependence of the couplings is well reproduced by a massive propagator with the mass $m=0.8$ in unit of $b$. The second is the $S U(3)$ monopole action recently obtained. The third is new interesting gauges showing abelian and monopole dominances as in the maximally abelian gauge.

\section{Detailed analyses of $S U(2)$ monopole ac- tion}

Confinement phenomena seem to be well reproduced by abelian link fields alone in the maximally abelian (MA) gauge in QCD 1 -5 5]. The abelian dominance suggests the existence of an effective $U(1)$ theory $S_{\text {eff }}(u)$ describing confinement.

After the abelian projection, one can separate out abelian link fields $u(s, \mu)$ as

$U^{\prime}(s, \mu)=V(s) U(s, \mu) V^{\dagger}(s+\hat{\mu}) \equiv c(s, \mu) u(s, \mu)$.

Shiba and one of the authors (T.S.)[6] determined a monopole effective action defined as [7]

$$
\begin{aligned}
\exp (-S[k]) & =\int D u \delta(k, u) \exp \left(-S_{\text {eff }}(u)\right) \\
\delta(k, u) & \equiv \delta\left(k_{\mu}(s)-\frac{1}{2} \epsilon_{\mu \nu \rho \sigma} \partial_{\nu} m_{\rho \sigma}(s+\hat{\mu})\right)
\end{aligned}
$$

performing a dual transformation numerically. They also considered $n^{3}$ extended monopoles defined on a sublattice with the spacing $b=n a$ [.

\footnotetext{
*The authors of each part are 1. T.Suzuki, S.Kitahara, N.Nakamura, M.Sei and S.Kato, 2. T.Suzuki, Y.Matsubara, N.Arasaki, S.Ejiri and S.Kitahara, 3. T.Suzuki and F.Shoji.
}

This corresponds to making a block-spin transformation on the dual lattice as seen from

$$
\begin{aligned}
e^{-S^{(n)}\left[k^{(n)}\right]}= & \left(\prod_{s, \mu} \sum_{k_{\mu}(s)=-\infty}^{\infty}\right)\left(\prod_{s} \delta_{\partial_{\mu}^{\prime} k_{\mu}(s), 0}\right) \\
\times \quad & \prod_{s, \mu} \delta\left(k_{\mu}^{(n)}(s)-F(k)\right) e^{-S[k]},
\end{aligned}
$$

$F(k)=\sum_{i, j, l=0}^{n-1} k_{\mu}(n s+(n-1) \hat{\mu}+i \hat{\nu}+j \hat{\rho}+l \hat{\sigma})$.

The monopole action adopted was composed of 12 two-point current-current interactions $S[k]=$ $\sum_{i} G_{i} S_{i}[k]$, the first of which is the self-coupling term $S_{1}[k]=\sum k_{\mu}^{2}(s)$. The effective monopole actions $S^{(n)}\left[k^{(n)}\right]$ for $n=1 \sim 4$ were fixed from the emsemble $\left\{k_{\mu}^{(n)}(s)\right\}$ calculated from vacuum configurations on $24^{4}$ lattice by extending the Swendsen method[9] for $\beta=2.5 \sim 2.8$. The monopole condensation [10] is shown to occur from energy-entropy balance 11. However, the vacuum configurations for such $\beta$ have large finite-size effects and the couplings $G_{i}(i \geq 2)$ have large errors.

It is the aim of this report to present new data for stronger coupling regions $\beta=2.0 \sim 2.5$. Since 
2

the data are surprisingly clearer, we have adopted a monopole action with 32 two-point couplings. The results are summarized as follows:

1. The couplings up to the distance $\sqrt{8}$ are fixed clearly.

2. The distance dependence of the couplings is well reproduced by a massive propagator with the mass $m=0.8$ in unit of $b$. The Smit-Sijs form (self-mass + Coulomb) is not good enough. See Fig. 1.

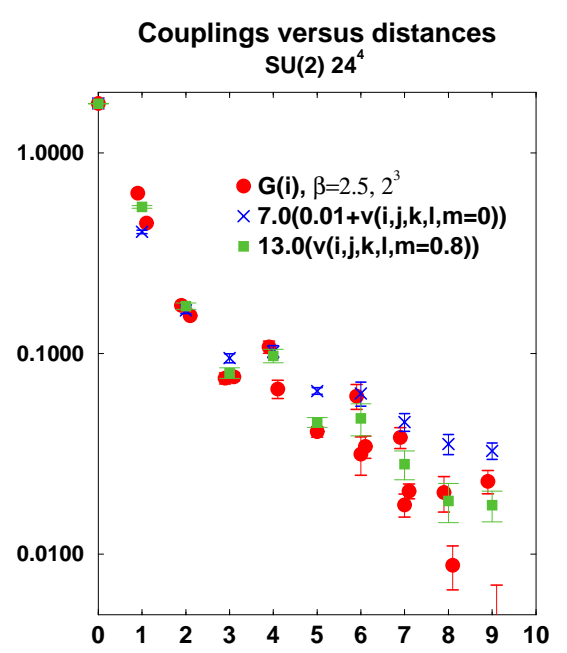

Figure 1. Couplings versus squared distance between two currents.

3. The scaling for fixed $b=n a(\beta)$ looks good for extendedness $n \geq 3$.

4. The $b$ dependence for all couplings are well fitted with a universal curve. See Fig 2 and also an example in Fig. 3.

5. The action is approximately fitted by the following form:

$$
\begin{aligned}
S(k) & =g(b) \sum_{i} k_{\mu}(s) \Delta^{-1}\left(\bar{i}, m^{2}\right) k_{\mu}(s+\bar{i}) \\
g(b) & =C \log \left(1+1 /(b \Lambda)^{2}\right) \\
m & \sim 0.8
\end{aligned}
$$

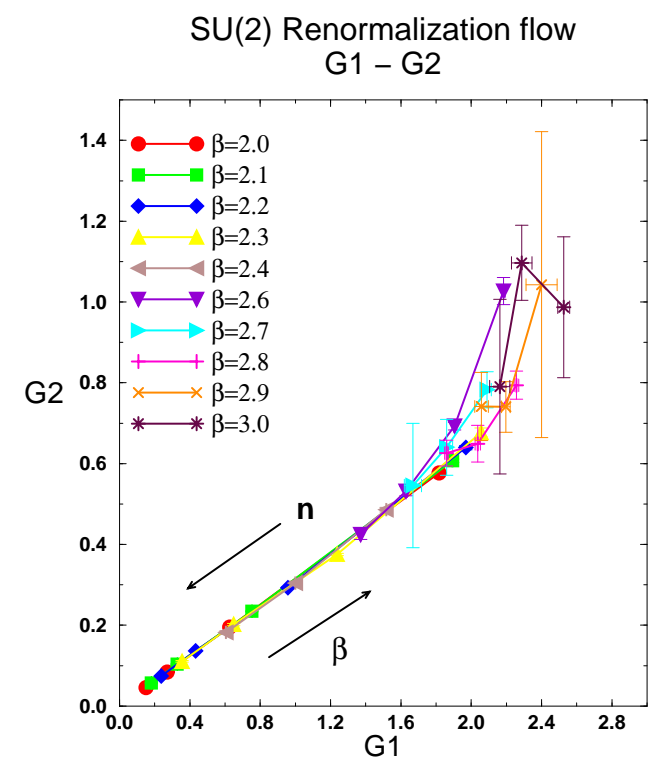

Figure 2. The $G_{1}-G_{2}$ cross section of the renormalization flow of the block-spin transformation on the dual lattice.

$$
\begin{aligned}
\Lambda & \sim 39 \Lambda_{L}, \\
C & \sim 4.9 .
\end{aligned}
$$

\section{The results of $S U(3)$ monopole action}

In the case of $S U(3)$, there are two independent (three with one constraint $\sum_{i=1}^{3} k_{\mu}^{i}(s)=0$ ) currents. When considering the two independent currents, their entropies are difficult to evaluate. Hence we first try to evaluate the effective monopole action, paying attention to only one monopole current.

The monopole action in $S U(3)$ QCD is obtained for $\beta=5.0 \sim 6.012,6$. Lattice sizes considered are $8^{4} \sim 24^{4}$ (for $T=0$ system). The results are summarized as follows:

1. The monopole actions for all extended monopoles are fixed in a compact form even in the scaling region.

2. Lattice-volume dependence is small.

3. The total action is well approximated by the product of the self-coupling constant and the length $f_{1} \times L$. 
Coupling constant versus $b$ G11(b)

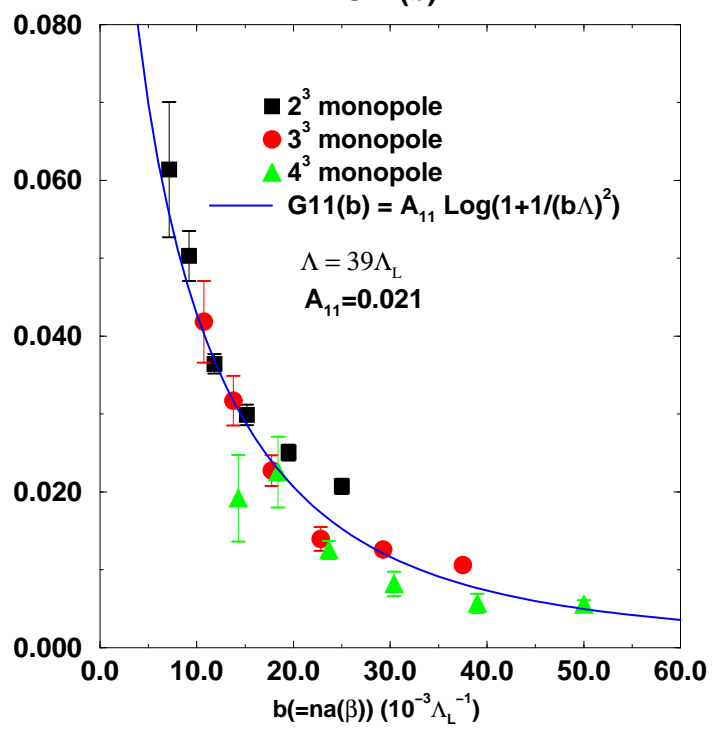

Figure 3. The $b=n a(\beta)$ dependence of the coupling $G_{11}$ in $S U(2)$.

4. Energy-entropy shows monopole condensation as seen in Fig. 4 .

5. Scaling is not yet seen in $S U(3)$.

6. There seems to be an infrared fixed-point at $f_{i}=0$ as in $S U(2)$.

\section{New gauges showing abelian and monopole dominance}

We have found two new gauges showing abelian and monopole dominances as in the maximally abelian (MA) gauge. The first one is the minimal abelian action (mAA) gauge which minimizes the abelian action:

$$
\begin{aligned}
R & =\sum_{s, \mu, \nu} \cos \theta_{\mu \nu}(s), \\
\theta_{\mu \nu}(s) & =\theta_{\mu}(s)+\theta_{\nu}(s+\mu)-\theta_{\mu}(s+\nu)-\theta_{\nu}(s) .
\end{aligned}
$$

When we take the $a \rightarrow 0$ continuum limit, we get

$$
\sum_{\mu, \nu} \partial_{\nu} f_{\mu \nu}(x) A_{\mu}^{ \pm}(x)=0 .
$$

The second is the minimal abelian monopole density (mAMD) gauge which minimizes the

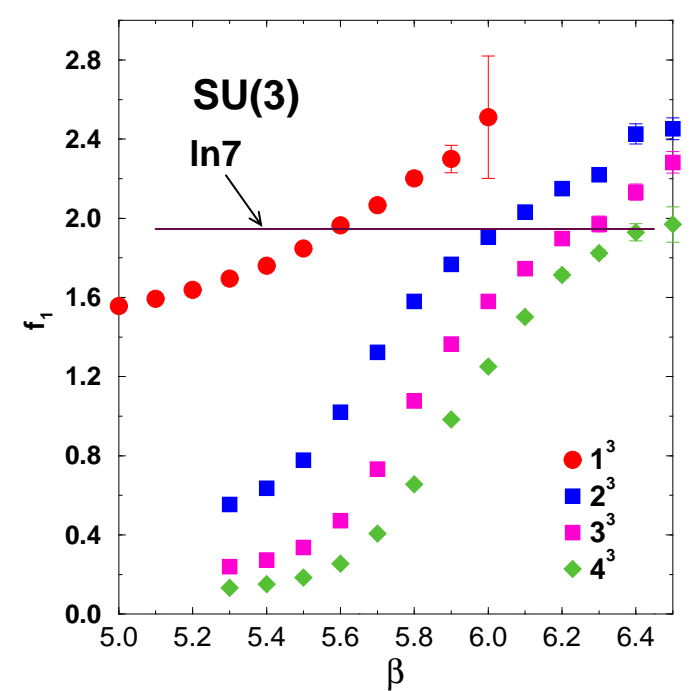

Figure 4. The $\beta$ dependence of the self-coupling term $f_{1}$ in $S U(3)$ case.

abelian monopole density:

$$
\begin{aligned}
\rho & =\sum_{s, \mu}\left|k_{\mu}(s)\right| . \\
k_{\mu}(s) & =-\frac{1}{2} \epsilon_{\mu \nu \alpha \beta} \partial_{\nu} \bar{\theta}_{\alpha \beta}(s+\mu) \\
\theta_{P} & =\bar{\theta}_{P}+2 \pi n_{P}
\end{aligned}
$$

We get the following:

1. Abelian Wilson loops in mAA are enhanced as in MA, but they are smaller in mAMD.

2. The string tension is reproduced by the abelian part and the monopole part as seen in Fig. 司.

3. The monopole densities in both new gauges are smaller than that in MA. See Fig. 6 .

4. The off-diagonal components in both gauges are less suppressed than in MA. See for example Fig. 7.

5. Almost the same monopole actions are obtained in MA and mAA.

The details of these three topics will be published elsewhere. 


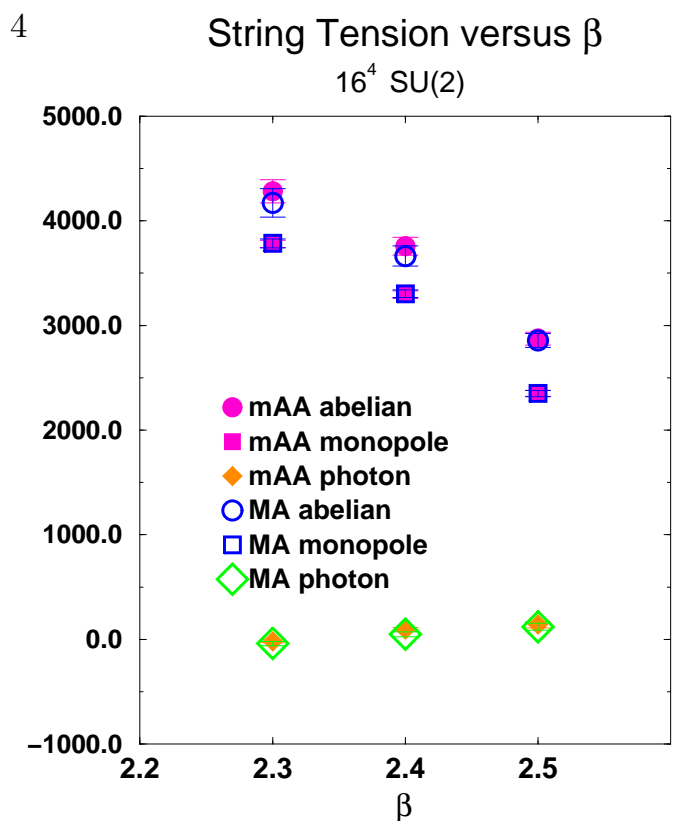

Figure 5. The string tension from the abelian , the monopole and the photon parts.

This work is financially supported by JSPS Grant-in Aid for Scientific Research (B) (No.06452028).

\section{REFERENCES}

1. A.S. Kronfeld et al., Phys. Lett. 198B, 516 (1987),

A.S. Kronfeld et al., Nucl.Phys. B293, 461 (1987).

2. T. Suzuki and I. Yotsuyanagi, Phys. Rev. D42, 4257 (1990); Nucl. Phys. B(Proc. Suppl.) 20, 236 (1991).

3. S. Hioki et al., Phys. Lett. 272B, 326 (1991).

4. T. Suzuki, Nucl. Phys. B(Proc. Suppl.) 30, 176 (1993) and references therein.

5. T. Suzuki, Talk at 'QCD on Massively Parallel Computers' held at Yamagata, Japan from March 16 till March 18, 1995.

6. H.Shiba and T.Suzuki, Phys. Lett. B351, 519 (1995) and references therein.

7. T.A. DeGrand and D. Toussaint, Phys. Rev. D22, 2478 (1980).

8. T.L. Ivanenko et al., Phys. Lett. 252B, 631 (1990).

9. R.H. Swendsen,Phys. Rev. Lett. 52,1165 (1984); Phys. Rev. B30, 3866, 3875 (1984). monopole density (Number/volume)

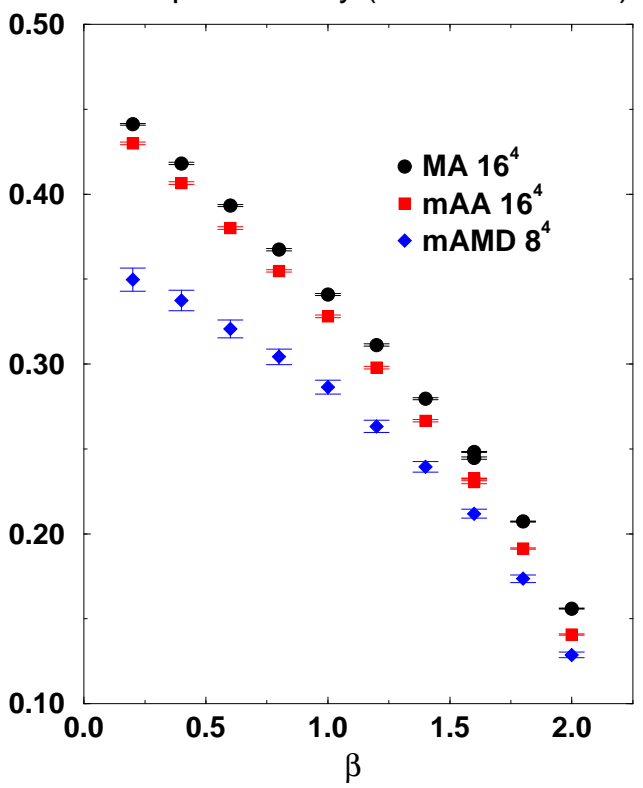

Figure 6. The monopole density in new gauges in comparison with that in MA.

10. G. 'tHooft, Nucl. Phys. B190, 455 (1981).

11. T.Banks et al., Nucl. Phys. B129, 493 (1977).

12. T.Suzuki et al., Nucl. Phys. B(Proc.Suppl.) 47, 270 (1995). 
Histogram of $|\mathrm{C}| \beta=2.1$

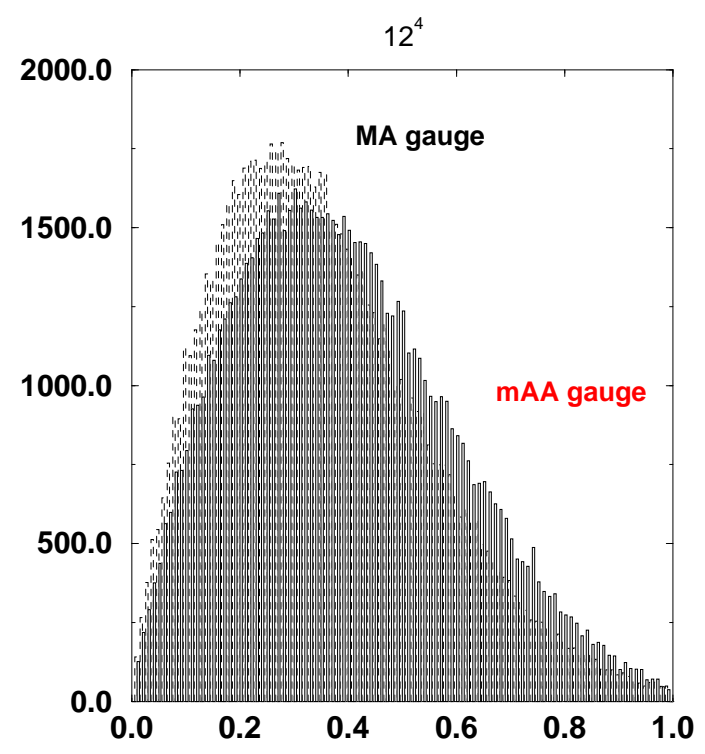

Figure 7. The histogram of the absolute value of the off-diagonal part in MA and mAA. 